\title{
Acquired perforating dermatosis in renal dialysis and diabetic patient: A case report
}

\author{
Meryem Khalidi, Rachid Frikh, Naoufal Hjira, Mohammed Boui
}

\author{
Dermatology-Venerology Department, Mohammed V Military Hospital of Instruction, Rabat, Morroco
}

Corresponding author: Meryem Khalidi, MD, E-mail: drkhalidimeryem@gmail.com

\begin{abstract}
Acquired perforating dermatosis (APD) is an uncommon disease characterized by lesions exhibiting transepidermal elimination of collagen or elastic fibers. APD affects adults and is associated with systemic diseases, mainly diabetes mellitus and renal failure. We report a new Moroccan case of this rare entity.

It's about a 62-year-old patient, followed for diabetes mellitus and renal failure under dialysis and who presented an extremely itchy rash, made of nodules with a keratotic center and associated with multiple scratching lesions.

The skin biopsy showed a perforated epidermis with the removal of material containing keratin and collagen.

the outcome was favorable with oral corticosteroids with a follow-up of one year.

APD is a rare dermatosis that affect considerably the quality of life of patients. its treatment remains suspensive pending the treatment of the cause.
\end{abstract}

Key words: Acquired perforating dermatosis; Diabetes mellitus; Chronic renal failure

\section{INTRODUCTION}

Perforating dermatoses are a group of conditions characterized by transepidermal elimination of dermal material (collagen, elastic tissue or necrotic connective tissue). Major perforating disorders include reactive perforating collagenosis (inherited disorder of collagen perforation), elastosis perforans serpiginosa (elastic tissue perforation associated with Down syndrome and Ehlers-Danlos syndrome), perforating folliculitis (perforation of necrotic material secondary to local trauma) and acquired perforating dermatosis .

Acquired perforating dermatosis (APD) is a rare disease that occurs in adults in many diseases such as diabetes, chronic kidney disease, lymphomas, solid tumors or certain infectious diseases.

We report a new observation of a patient who present APD associated to diabetes and chronic renal failure.

\section{CASE REPORT}

It's about a 62-year-old patient who was referred to us for treatment of an extremely itchy rash that invalidates the patient's daily life. In his antecedents, we found a chronic diabetes evolving for 20 years complicated by end-stage renal failure in the hemodialysis stage, hypertension and cured hepatitis $\mathrm{C}$. The medical examination objectified nodules with a depressed keratotic center giving them an umbilical appearance scattered throughout the integument (Figs. 1 - 3) and associated with intense scratching lesions (Fig. 4). The biological assessment revealed a slight inflammatory syndrome. The skin biopsy showed a perforated epidermis with the removal of material containing keratin and collagen (Fig. 5). The patient was put on oral corticosteroid therapy at $0.5 \mathrm{mg} / \mathrm{kg}$ with almost complete improvement of the lesions. The follow-up is 1 year. corticosteroid therapy is always maintained at a lower dose. Phototherapy and cyclines were ineffective.

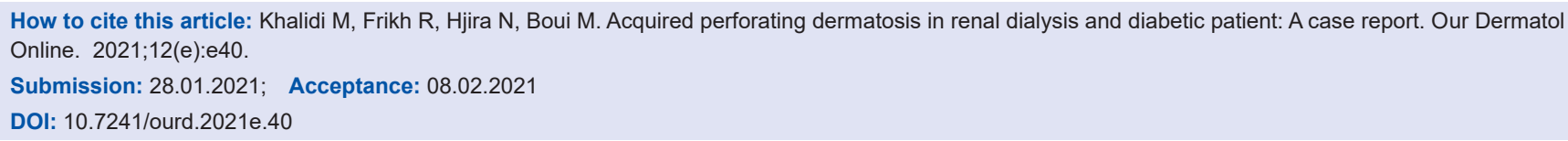




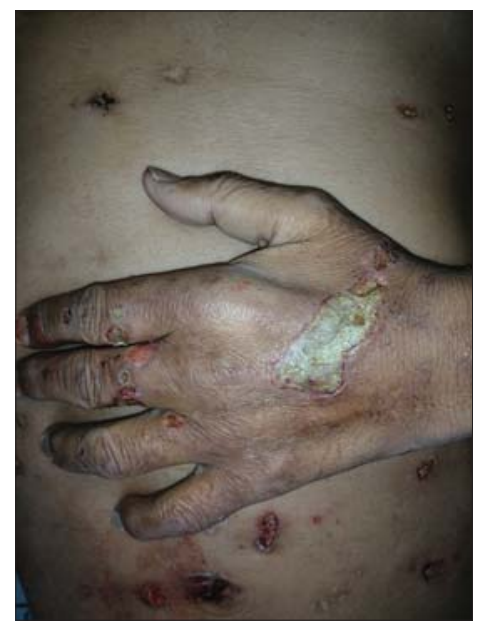

Figure 1: A large keratotic nodule of the left hand.

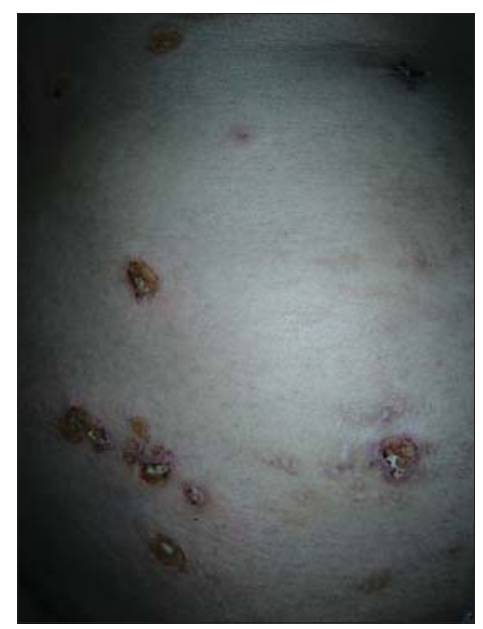

Figure 2: Umbilical and depressed keratotic nodules.

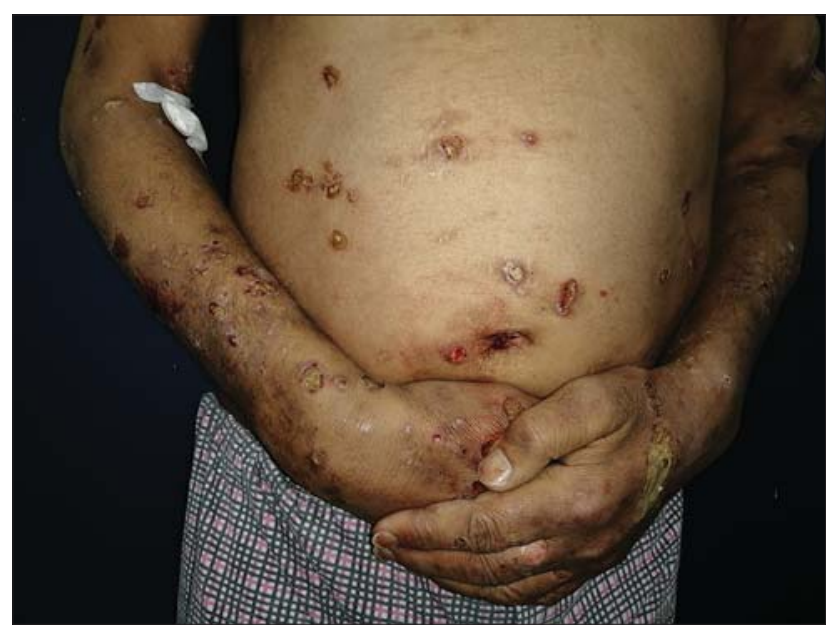

Figure 3: Nodular keratotic and itchy eruption of arms and abdomen.

\section{DISCUSSION}

The perforating dermatoses are a heterogeneous group of diseases characterized by the transepidermal

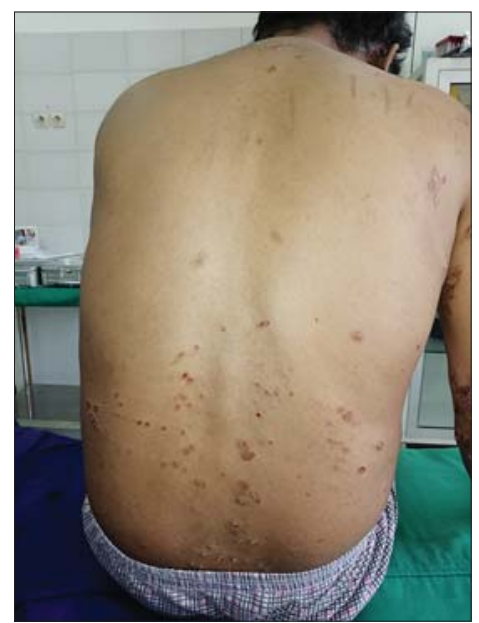

Figure 4 : Intense scratching marks.

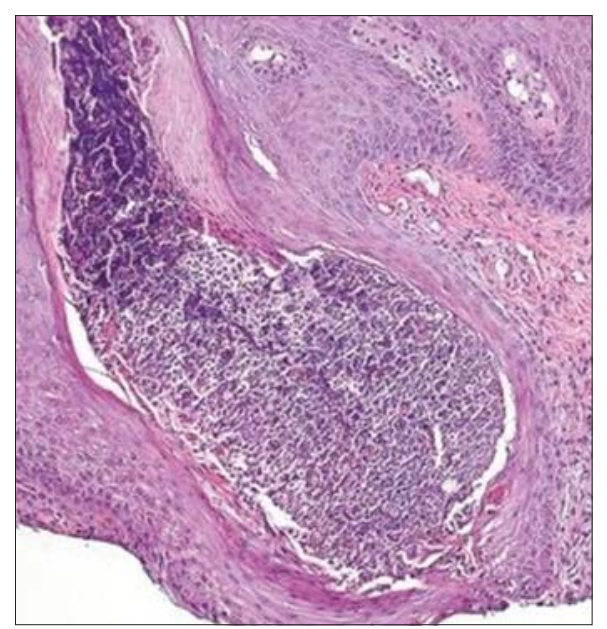

Figure 5 : Perforation of epidermis with removal of material containing keratin and collagen.

elimination of one or more components of the skin. Four types are traditionally recognized: elastosis perforans serpiginosum, perforating folliculitis, Kyrle disease, and reactive perforating collagenosis (RPC). Two variants of RPC have been described: a rare hereditary form that presents in childhood and a more common acquired form that appears in adult life and mainly affects diabetic patients with chronic renal failure (CRF). Over the years, there has been a degree of confusion regarding the terminology. At the present time, the majority of authors use the term reactive perforating collagenosis for the hereditary form, while the term acquired perforating dermatosis (APD), is preferred for the adult form and for any perforating disease that is clinically and histologically similar to the primary disease but that is associated with a systemic disease [1].

Acquired perforating dermatosis is a rare skin complication in diabetes and chronic kidney 
disease. It's pathophysiology in diabetes has been elucidated: in fact, hyperglycemia alters structural proteins and results in the production of terminal glycation products [2]. This results in glycation of the extracellular matrix, which causes the production by keratinocytes of involucrin, a molecule that promotes their expulsion as well as that of collagen through the epidermis [3].

It classically presents with severely pruritic follicular hyperkeratotic papules, sometimes umbilicated, on the hair-bearing limbs of adults, as in our patient. Generalized papules may also be seen. APD is a chronic disease, usually associated with diabetes mellitus or renal failure or both [4]. In patients receiving dialysis, acquired perforating dermatosis occurs in about $10 \%$ of patients. It is also rarely associated with liver disease, malignant disease, hypothyroidism and HIV [5].

The differential diagnosis includes prurigo nodularis, folliculitis, arthropod bites, multiple keratoacanthomas, psoriasis and lichen planus [4].

Investigations include skin biopsy and a search for associated systemic diseases. Treatment may be directed at relieving pruritus, because perforating disorders can be exacerbated by koebnerization (lesions appearing at sites of minor trauma, as in psoriasis). Most treatment strategies are supported by anecdotal evidence and include oral or topical retinoids and topical or intralesional corticosteroids. Phototherapy (narrow-band or broad-band UVB, or psoralen plus UVA) may be helpful for pruritus [6]. Other approaches include the use of antibiotics (e.g., doxycycline) and destructive methods such as cryotherapy, surgical debridement and laser therapy. Various other local treatments are offered: emollient creams, capsaicin, tretinoin., isotretinoin, rifampicin, allopurinol have also been offered with varying results. The condition progresses favorably after kidney transplantation [7].

\section{CONCLUSION}

APD is one of the dermatological manifestations of certain chronic conditions including diabetes and chronic kidney disease. its diagnosis and treatment are not easy and the quality of life of patients is often severely affected.

\section{Consent}

The examination of the patient was conducted according to the principles of the Declaration of Helsinki.

The authors certify that they have obtained all appropriate patient consent forms, in which the patients gave their consent for images and other clinical information to be included in the journal. The patients understand that their names and initials will not be published and due effort will be made to conceal their identity, but that anonymity cannot be guaranteed.

\section{REFERENCES}

1. Metterle L, Magro CM, Zang JB. Giant variant of acquired perforating dermatosis in a renal dialysis patient. JAAD Case Rep. 2017;3:42-4.

2. Saray Y, Seçkin D, Bilezikçi B. Acquired perforating dermatosis: clinicopathological features in twenty-two cases. J Eur Acad Dermatol Venereol. 2006;20:679-88.

3. Hong SB, Park JH, Ihm CG, Kim NI. Acquired perforating dermatosis in patients with chronic renal failure and diabetes mellitus. J Korean Med Sci. 2004;19:283-8.

4. Rapini RP, Hebert AA, Drucker CR. Acquired perforating dermatosis. Arch Dermatol. 2011;125:1074-8.

5. Tiwary AK, Mishra DK, Chaudhary SS. A rare case of familial reactive perforating collagenosis. Our Dermatol Online. 2017;8:170-3.

6. Imam TH, Patail H, Khan N, Hsu PT, Cassarino DS. Acquired perforating dermatosis in a patient on peritoneal dialysis: a case report and review of the literature. Case Rep Nephrol. 2018;2018:5953069.

7. González-Lara L, Gómez-Bernal S, Vázquez-López F, VivancoAllende B. Acquired perforating dermatosis: a report of 8 cases. Dermatology (Actas Dermo-Sifiliográficas, English Edition). 2014;105:e39-e43,

Copyright by Meryem Khalidi, et al. This is an open access article distributed under the terms of the Creative Commons Attribution License, which permits unrestricted use, distribution, and reproduction in any medium, provided the original author and source are credited.

Source of Support: Nil, Conflict of Interest: None declared. 